University of New Orleans

ScholarWorks@UNO

6-1992

\title{
Limaçon of Pascal locus of the complex refractive indices of interfaces with maximally flat reflectance-versus-angle curves for incident unpolarized light
}

R. M.A. Azzam

University of New Orleans, razzam@uno.edu

Follow this and additional works at: https://scholarworks.uno.edu/ee_facpubs

Part of the Electrical and Electronics Commons, and the Optics Commons

\section{Recommended Citation}

R. M. A. Azzam, "Limaçon of Pascal locus of the complex refractive indices of interfaces with maximally flat reflectance-versus-angle curves for incident unpolarized light," J. Opt. Soc. Am. A 9, 957-563 (1992)

This Article is brought to you for free and open access by the Department of Electrical Engineering at ScholarWorks@UNO. It has been accepted for inclusion in Electrical Engineering Faculty Publications by an authorized administrator of ScholarWorks@UNO. For more information, please contact scholarworks@uno.edu. 


\title{
Limaçon of Pascal locus of the complex refractive indices of interfaces with maximally flat reflectance-versus-angle curves for incident unpolarized light
}

\author{
R. M. A. Azzam \\ Department of Electrical Engineering, University of New Orleans, Lakefront, New Orleans, Louisiana 70148
}

Received March 25, 1991; revised manuscript received December 19, 1991; accepted December 26, 1991

\begin{abstract}
For an interface between two isotropic media the power reflectance $R_{\nu}(\phi)$ is an even function, $R_{\nu}(\phi)=R_{\nu}(-\phi)$, of the angle of incidence $\phi$; hence all the odd derivatives, $R_{\nu}{ }^{(n)}=d^{n} R_{\nu} / d \phi^{n}(n$ odd), are identically 0 at $\phi=0$, independent of the incident polarization $\nu$. When the incident light is unpolarized $(\nu=u)$, the second derivative, $R_{u}{ }^{(2)}$, is also 0 at $\phi=0$, so that the flatness of the $R_{u}$-versus- $\phi$ curve over an initial range of $\phi$ starting from $\phi=0$ is determined by the fourth derivative, $R_{u}{ }^{(4)}$. The condition that $R_{u}{ }^{(4)}=0$ at $\phi=0$ gives the maximally flat response and leads to a specific constraint on the complex relative refractive index $N$, namely, that $\operatorname{Re}\left[(N-1)^{2} / N^{3}\right]=2 / N N^{*}$. The corresponding complex plane contour is the limaçon of Pascal, $\eta=2 \cos \theta \pm$ $\sqrt{3}$ in polar form, where $N=\eta \exp (j \theta)$. The two branches of this contour constitute the boundary lines that separate the region of the complex plane in which the function $R_{u}(\phi)$ is monotonic from that in which the function exhibits a minimum at oblique incidence. Families of curves that illustrate the maximally flat response in external and internal reflection are presented. New equations that determine the angle of incidence of minimum unpolarized-light reflectance of a dielectric-dielectric or a dielectric-conductor interface are derived.
\end{abstract}

\section{INTRODUCTION}

The power (or intensity) reflectance of a planar interface between a transparent medium of incidence and an absorbing medium of refraction for incident unpolarized or circularly polarized light is a function of the angle of incidence $\phi$ and the wavelength-dependent complex relative refractive index $N$. (It is assumed that the incident light is collimated and monochromatic or quasi-monochromatic and that the two media are homogeneous, optically isotropic, linear, and nonmagnetic.) This reflectance $R_{u}$ is the average,

$$
R_{u}=\left(R_{p}+R_{s}\right) / 2,
$$

of the power reflectances $R_{p}$ and $R_{s}$ for the $p$ and the $s$ linear polarizations, parallel and perpendicular to the plane of incidence, respectively. The latter are calculated from

$$
R_{p}=r_{p} r_{p}^{*}, \quad R_{s}=r_{s} r_{s}^{*},
$$

where $r_{p}$ and $r_{s}$ are the complex-amplitude Fresnel reflection coefficients given by ${ }^{1}$

$$
\begin{aligned}
r_{p} & =\left(N^{2} \cos \phi-X\right) /\left(N^{2} \cos \phi+X\right), \\
r_{s} & =(\cos \phi-X) /(\cos \phi+X), \\
X & =\left(N^{2}-\sin ^{2} \phi\right)^{1 / 2}
\end{aligned}
$$

From Eqs. (3) it is readily apparent that $r_{\nu}$ is an even function of $\phi$, i.e.,

$$
r_{\nu}(\phi)=r_{\nu}(-\phi), \quad \nu=p, s .
$$

Changing the sign of $\phi$ is permitted mathematically and may be thought of physically as changing the direction of incidence from one side of the interface normal to the other in a given plane of incidence. From Eqs. (1) and (2) it follows that the power reflectances $R_{p}, R_{s}$, and $R_{u}$ are also even functions of $\phi$ and can be expressed in terms of their Taylor-MacLaurin-series expansion as

$$
\begin{aligned}
R_{\nu}=R_{\nu}(0)+R_{\nu}^{\prime \prime}(0)\left(\phi^{2} / 2 !\right)+R_{\nu}^{\prime \prime \prime}(0)\left(\phi^{4} / 4 !\right) & +\ldots, \\
\nu & =p, s, u .
\end{aligned}
$$

The second derivatives $R_{\nu}{ }^{\prime \prime}$ were evaluated previously, ${ }^{2}$ and it was found that $R_{p}^{\prime \prime}(0)=-\mathrm{R}_{\mathrm{s}}^{\prime \prime}(0)$, so that

$$
R_{u}{ }^{\prime \prime}(0)=0 \text {. }
$$

Therefore the initially parabolic (or quadratic) rise of $R_{s}$ with $\phi$ is exactly compensated, or balanced, by the initially parabolic fall of $R_{p}$, which makes the unpolarizedlight (or average) reflectance $R_{u}$ substantially flat with respect to $\phi$ near $\phi=0$. From Eqs. (5) and (6) the angular variation of $R_{u}$ is determined by

$$
R_{u}=R_{u}(0)+R_{u}{ }^{\prime \prime \prime}(0)\left(\phi^{4} / 4 !\right)+\ldots
$$

This paper concerns the constraint on complex $N$ such that the fourth derivative of $R_{u}$ is also 0 at normal incidence, i.e.,

$$
R_{u}^{\prime \prime \prime \prime}(0)=0 .
$$

This leads to the maximally flat reflectance (MFR) $R_{u}$ versus- $\phi$ curve that is described by a series in which the leading $\phi$-dependent term is of the sixth power,

$$
R_{u}=R_{u}(0)+R_{u}^{(6)}(0)\left(\phi^{6} / 6 !\right)+\ldots,
$$

since all the first five derivatives are zero. The flatness of the curve is best appreciated through the specific examples to be presented. Surfaces with a flat reflectanceversus-angle curve can be useful in scanning applications 
in which a light beam is to be deflected over a wide range of angles without introducing any significant changes of the reflected light intensity.

\section{EVALUATION OF THE FOURTH DERIVATIVES AT NORMAL INCIDENCE}

For simplicity, the derivatives of the complex Fresnel reflection coefficients $r_{p}$ and $r_{s}$ are determined first. By carrying out two successive differentiations of the already published second derivatives ${ }^{2}$ and evaluating the results at $\phi=0$, we obtain

$$
\begin{aligned}
& r_{p}^{\prime \prime \prime \prime}(0)=-2 r_{p}(0)\left(5 N^{2}-6 N+9\right) / N^{3}, \\
& r_{s}^{\prime \prime \prime \prime}(0)=-2 r_{s}(0)\left(N^{2}-6 N-3\right) / N^{3} .
\end{aligned}
$$

For completeness I also list

$$
\begin{aligned}
r_{p}{ }^{\prime \prime}(0) & =-2 r_{p}(0) / N \\
r_{s}{ }^{\prime \prime}(0) & =2 r_{s}(0) / N \\
r_{p}(0) & =-r_{s}(0)=(N-1) /(N+1) .
\end{aligned}
$$

The derivatives of a power reflectance $R_{\nu}(\nu=p$ or $s)$ are related to those of the associated complex Fresnel reflection coefficient $r_{\nu}$ by

$$
\begin{aligned}
R_{\nu}{ }^{\prime \prime} & =2 \operatorname{Re}\left(r_{\nu}{ }^{\prime \prime} r_{\nu}{ }^{*}+r_{\nu}{ }^{\prime} r_{\nu}{ }^{*}\right), \\
R_{\nu}{ }^{\prime \prime \prime \prime} & =2 \operatorname{Re}\left(r_{\nu}{ }^{\prime \prime \prime \prime} r_{\nu}{ }^{*}+4 r_{\nu}{ }^{\prime \prime \prime} r_{\nu}{ }^{*}+3 r_{\nu}{ }^{\prime \prime} r_{\nu}{ }^{\prime \prime *}\right)
\end{aligned}
$$

at any angle of incidence $\phi$. At normal incidence, $\phi=0$, the odd derivatives vanish, and Eqs. (15) and (16) simplify to

$$
\begin{aligned}
R_{\nu}{ }^{\prime \prime}(0) & =2 \operatorname{Re}\left[r_{\nu}{ }^{\prime \prime}(0) r_{\nu}(0)^{*}\right], \\
R_{\nu}^{\prime \prime \prime \prime}(0) & =2 \operatorname{Re}\left[r_{\nu}^{\prime \prime \prime \prime}(0) r_{\nu}(0)^{*}+3 r_{\nu}{ }^{\prime \prime}(0) r_{\nu}{ }^{\prime \prime}(0)^{*}\right]
\end{aligned}
$$

The fourth derivative of $R_{u}$ is

$$
R_{u}^{\prime \prime \prime \prime}(0)=\left[R_{p}^{\prime \prime \prime \prime}(0)+R_{s}^{\prime \prime \prime \prime}(0)\right] / 2,
$$

and the condition for the MFR of Eq. (8) can be restated as

$$
R_{p}^{\prime \prime \prime \prime}(0)+R_{s}^{\prime \prime \prime \prime}(0)=0 \text {. }
$$

\section{CONSTRAINT ON THE COMPLEX REFRACTIVE INDEX $N$ FOR MAXIMALLY FLAT UNPOLARIZED-LIGHT REFLECTANCE}

From Eqs. (10)-(13) and (17)-(19) we determine that

$$
R_{u}^{\prime \prime \prime \prime}(0)=24 R_{0}\left(1 / N N^{*}\right)-12 R_{0} \operatorname{Re}\left[(N-1)^{2} / N^{3}\right],
$$

where $R_{0}$ is the normal-incidence reflectance:

$$
\begin{aligned}
R_{0} & =r_{p}(0) r_{p}{ }^{*}(0)=r_{s}(0) r_{s}^{*}(0) \\
& =\left[N N^{*}-2 \operatorname{Re}(N)+1\right] /\left[N N^{*}+2 \operatorname{Re}(N)+1\right] .
\end{aligned}
$$

Setting $R_{u}{ }^{\prime \prime \prime}(0)$ of Eq. (21) equal to 0 gives the constraint on complex $N$ for the MFR:

$$
\operatorname{Re}\left[(N-1)^{2} / N^{3}\right]=\left(2 / N N^{*}\right) .
$$

Substitution of $N$ in the standard form

$$
N=n+j k
$$

(where $n$ is the refractive index and $k$ is the extinction coefficient) into Eq. (23) yields

$$
n^{4}+2 n^{2} k^{2}+k^{4}-4 n k^{2}-4 n^{3}+n^{2}-3 k^{2}=0 .
$$

Equation (25) gives the Cartesian equation of the locus of all the points in the $n k$ plane that represent interfaces with the MFR-versus-angle curves for incident unpolarized light. The nature of this quartic curve is not readily apparent from Eq. (25). For a given $n$, Eq. (25) is a quadratic equation in $k^{2}$ with solutions given by

$$
k^{2}=\left[-\left(2 n^{2}-4 n-3\right) \pm(24 n+9)^{1 / 2}\right] / 2 .
$$

A more elegant and transparent equation for the locus is obtained when $N$ is expressed in the polar form

$$
N=\eta \exp (j \theta),
$$

where

$$
\eta=\left(n^{2}+k^{2}\right)^{1 / 2}, \quad \theta=\arctan (k / n) .
$$

Substitution of $N$ from Eq. (27) into Eq. (23) leads to a quadratic equation in $\eta$ :

$$
\eta^{2}-4 \eta \cos \theta+\left(4 \cos ^{2} \theta-3\right)=0,
$$

with two solutions:

$$
\eta=2 \cos \theta \pm \sqrt{3} .
$$

Equation (30) is surprisingly simple and indicates that the locus of complex $N$ of all possible interfaces with the MFR is the limaçon of $\mathrm{Pascal}^{3}$ in the $n k$ plane. This locus is easily established by the graphic construction shown in Fig. 1. A semicircle is drawn with the center on the $n$ axis at $(1,0)$ and radius 1 ; the circle is tangent to the $k$ axis at the origin $\mathrm{O}$. The straight line $O P$ from the origin $\mathrm{O}$ to an arbitrary point $P$ on the circle, a cord of length $2 \cos \theta$ that makes an angle $\theta$ with the $n$ axis, is extended by a fixed amount $=\sqrt{3}$ to reach a point $Q$. The locus of $Q$,

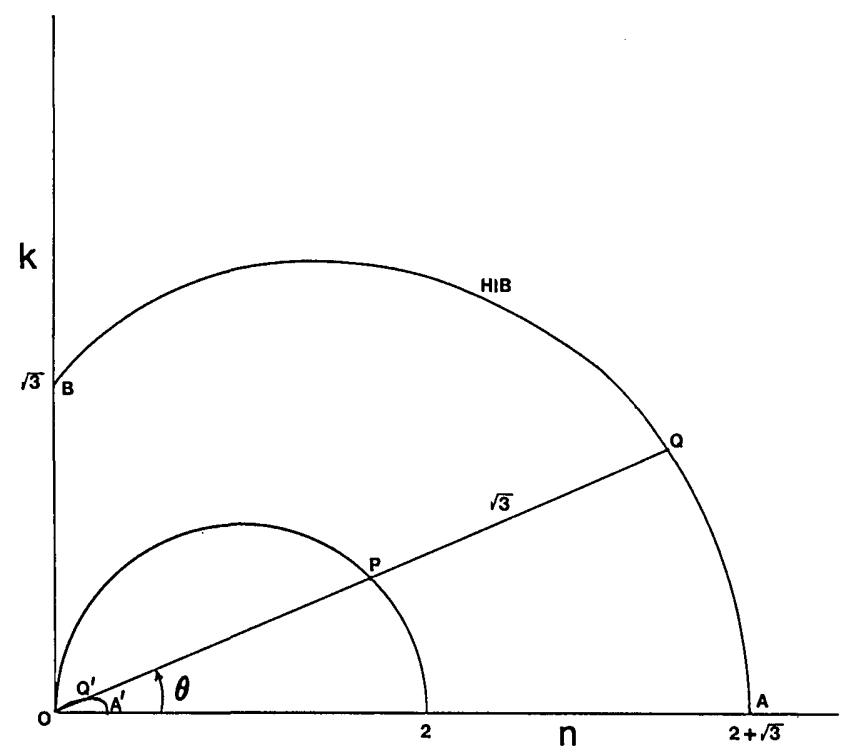

Fig. 1. Limaçon of Pascal locus of points that represent the complex relative refractive indices $N=(n, k)$ of all the possible interfaces with the MFR versus the angle of incidence for incident unpolarized or circularly polarized light. See text. 


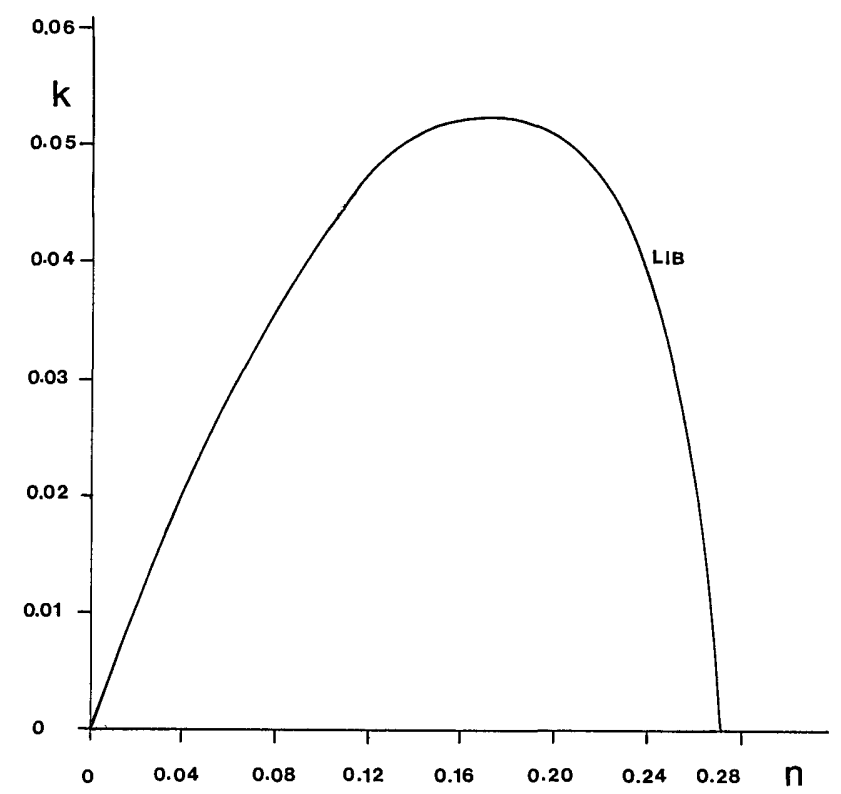

Fig. 2. Magnified view of the LIB of the limaçon of Pascal in Fig. 1.

as $\theta$ is increased from $0^{\circ}$ to $90^{\circ}$, is the limaçon of Pascal. This is the high-index branch (HIB) that corresponds to the + in Eq. (30).

If the - in Eq. (30) is chosen, a low-index branch (LIB) inside the semicircle is generated. It is the locus of a point $Q^{\prime}$ that is obtained by subtracting $\sqrt{3}$ from the length of the cord $O P$. Because negative values of $\eta$ are not permitted, the full LIB is traced as $\theta$ sweeps only the reduced range $0^{\circ} \leq \theta \leq 30^{\circ}$. The LIB lies entirely in the domain of fractional optical constants and is not sufficiently clear on the scale of Fig. 1 . Figure 2 shows the LIB on a magnified scale. The domain of fractional optical constants is of interest in the vacuum untraviolet and under conditions of internal reflection.

The finite area in the first quadrant of the $n k$ plane that is bounded by the HIB, the LIB, and the $n$ and the $k$ axes specifies the domain of the complex relative refractive indices of all the possible interfaces for which the unpolarized-light reflectance $R_{u}$ increases monotonically with the angle of incidence $\phi$. For any complex $N$ outside this domain, $R_{u}$ exhibits a minimum at oblique incidence. Previous attempts to define the regions of the $n k$ plane of monotonic and nonmonotonic behaviors were only partially successful. In an extensive paper by $\mathrm{Holl}^{4}$ the HIB is illustrated in a graph with an accompanying word of caution with regard to accuracy. Azzam and Alnajjar ${ }^{5}$ presented the HIB as determined by brute-force but accurate numerical iteration. Both of these earlier nonanalytic studies missed the LIB entirely.

\section{SOME PROPERTIES OF THE LIMAÇON OF PASCAL LOCUS OF COMPLEX REFRACTIVE INDICES FOR MAXIMALLY FLAT REFLECTANCE}

From Eqs. (24), (27), and (30), the parametric equations of the locus are

$$
n=(2 \cos \theta \pm \sqrt{3}) \cos \theta, \quad k=(2 \cos \theta \pm \sqrt{3}) \sin \theta .
$$

Recall that $0^{\circ} \leq \theta \leq 90^{\circ}$ for the HIB and $0^{\circ} \leq \theta \leq 30^{\circ}$ for the LIB. Any pair $(n, k)$ calculated from Eqs. (31) represents an interface with a maximally flat $R_{u}$-versus- $\phi$ curve.

The points of intersection A and B of the HIB with the $n$ and the $k$ axes are located at

$$
N_{A}=(2+\sqrt{3})+j 0, \quad N_{B}=0+j \sqrt{3},
$$

respectively. The LIB intersects the same axes at the origin $O$ and at $\mathrm{A}^{\prime}$, where

$$
N_{A^{\prime}}=(2-\sqrt{3})+j 0 .
$$

Note that $N_{A} N_{A^{\prime}}=1$, so that A and $\mathrm{A}^{\prime}$ correspond to the external and the internal reflection, respectively, at the same dielectric-dielectric interface with relative refractive index $2+\sqrt{3}=3.73205$.

It is also of interest to locate the points on the HIB and the LIB at which the extinction coefficient $k$ is maximum. This is done by setting $\partial k / \partial \theta=0$, where $k$ is given by the second of Eqs. (31). This gives a quadratic equation in $\cos \theta$ with solutions $\cos \theta=0.5230$, hence $\theta=58.466^{\circ}$ on the HIB; and $\cos \theta=0.9560$, hence $\theta=17.056^{\circ}$ on the LIB. Therefore the points of maximum $k$ are

$$
\begin{aligned}
N=\left(n, k_{\max }\right) & =(1.4529,2.3678) & & \text { for the HIB, } \\
& =(0.1721,0.0528) & & \text { for the LIB } .
\end{aligned}
$$

An important conclusion is that the unpolarized-light reflectance exhibits a minimum at oblique incidence if either one of the following (sufficient but not necessary) conditions is satisfied:

$$
n>3.73205, \quad k>2.3678 .
$$

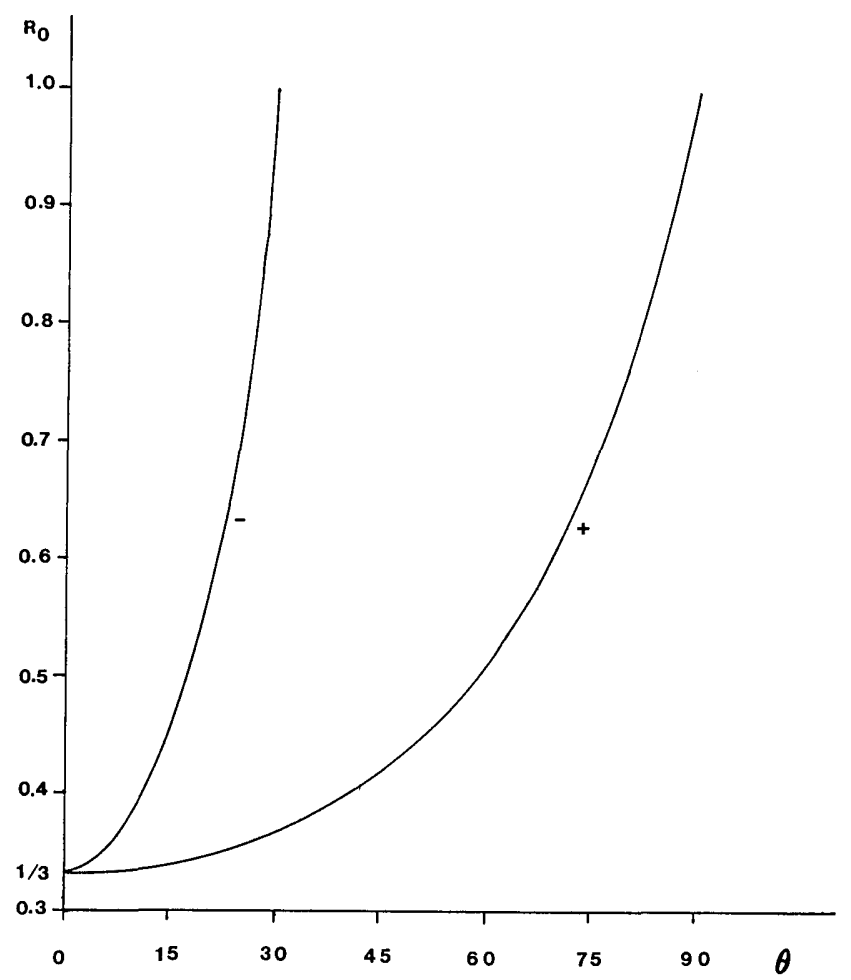

Fig. 3. Normal-incidence ref lectance $R_{0}$ of an interface with the MFR-versus-angle curve as a function of the angular polar coordinate $\theta$ on the limaçon of Pascal in Fig. 1. 
The variation of the normal-incidence reflectance $R_{0}$ as a function of position on the limaçon of Pascal is obtained from Eqs. (22), (27), and (30) as

$$
R_{0}=(2 \pm \sqrt{3} \cos \theta) /\left(4 \cos ^{2} \theta \pm 3 \sqrt{3} \cos \theta+2\right),
$$

where $\theta$ is the angular polar coordinate of a point on the contour as shown in Fig. 1 . As $\theta$ is increased from $0^{\circ}$ to $90^{\circ}$ on the HIB or from $0^{\circ}$ to $30^{\circ}$ on the LIB, $R_{0}$ increases monotonically from $1 / 3$ to 1 (Fig. 3 ). The rise of $R_{0}$ with $\theta$ is initially slow in the neighborhood of points $\mathrm{A}$ and $\mathrm{A}^{\prime}$ but becomes steep as points $\mathrm{B}$ and $\mathrm{O}$, respectively, are approached.

Any interface that has a normal-incidence reflectance $R_{0} \leq 1 / 3$ is characterized by a monotonic $R_{u}$-versus- $\phi$ curve. The corresponding range of $n$ and $k$ in Fig. 1 is bounded by the segment $A^{\prime} A$ of the $n$ axis and the semicircle constructed on $A^{\prime} A$ as a diameter; this range lies entirely between the LIB and the HIB.

Finally, all the possible values of the complex relative dielectric function $\epsilon$ for interfaces with the MFR are given by

$$
\begin{aligned}
& \epsilon=N^{2}, \\
& \epsilon=(2 \cos \theta \pm \sqrt{3})^{2} \exp (j 2 \theta),
\end{aligned}
$$

where the limits on $\theta$ are the same as noted above.

\section{MAXIMALLY FLAT REFLECTANCE CURVES}

Figure 4 shows a family of maximally flat $R_{u}$-versus- $\phi$ curves for 10 interfaces that correspond to 10 points on the HIB of the limaçon of Pascal at equispaced values of $\theta$ from $0^{\circ}$ to $90^{\circ}$ in equal steps of $10^{\circ}$. The curve marked a is for the dielectric-dielectric interface with $N=3.73205$. The extent to which $R_{u}$ remains virtually constant, $R_{u}=$ $R_{0}$, as $\phi$ is increased from 0 , is remarkable. For curve a, the reflectance increases from its normal-incidence value of $1 / 3$ by a scant 0.000006 as $\phi$ is increased from $0^{\circ}$ to $30^{\circ}$ (this is one third of the full range of $\phi$ ). Further increases of $\phi$ to $45^{\circ}$ and $60^{\circ}$ increase $R_{u}$ to 0.333544 and 0.336706 , respectively. Beyond $70^{\circ}$ the reflectance starts to rise steeply toward 1.
The reflection of red or near-infrared light at the air-Si interface is closely described by the MFR curve a, shown in Fig. 4. For example, at a wavelength of $747 \mathrm{~nm}, N=$ $3.736+j 0.009$ for $\mathrm{Si}^{6}{ }^{6}$ which is a good approximation to the required index. Coincidentally, the complex refractive index of GaAs at the same $747-\mathrm{nm}$ wavelength ${ }^{6}$ is $3.725+j 0.101$. A radial line from the origin to the point that represents the air-GaAs interface $\left(\theta=1.55^{\circ}\right)$ intersects the limaçon of Pascal at $3.730+j 0.101$. This differs little (by 0.005) from the GaAs index. A third case, which is also described sufficiently well by curve a of Fig. 4, is the air $-\mathrm{NaCl}$ interface with relative refractive index ${ }^{6} 3.72+j 0.02$ at the far-infrared wavelength of $71.43 \mu \mathrm{m}$.

For completeness, Fig. 5 shows a family of MFR curves under conditions of internal reflection at interfaces that are represented by points on the LIB branch of the limaçon of Pascal at $\theta=0^{\circ}, 5^{\circ}, \ldots, 30^{\circ}$, in equal steps of $5^{\circ}$.

\section{ANGLE OF INCIDENCE OF MINIMUM REFLECTANCE FOR INCIDENT UNPOLARIZED LIGHT}

When $N$ is such that the $R_{u}$-versus- $\phi$ curve exhibits a minimum at oblique incidence, it is of interest to locate the angular position of the minimum. This is done by finding $\phi$ such that

$$
R_{u}{ }^{\prime}(\phi)=0
$$

or, equivalently,

$$
\operatorname{Re}\left(r_{p}{ }^{\prime} r_{p}^{*}+r_{s}{ }^{\prime} r_{s}^{*}\right)=0 .
$$

When the Fresnel coefficients and their first derivatives are substituted into Eq. (40), the result can be reduced to the following form:

$$
\begin{aligned}
\operatorname{Re}\left[\epsilon^{*}\left(\epsilon \cos ^{2} \phi-\sin ^{2} \phi\right) X\right] / \operatorname{Re}(X) & \\
& =|(\epsilon \cos \phi+X) /(\cos \phi+X)|^{4},
\end{aligned}
$$

where $X=\left(\epsilon-\sin ^{2} \phi\right)^{1 / 2}$. For a given complex $\epsilon\left(=N^{2}\right)$,

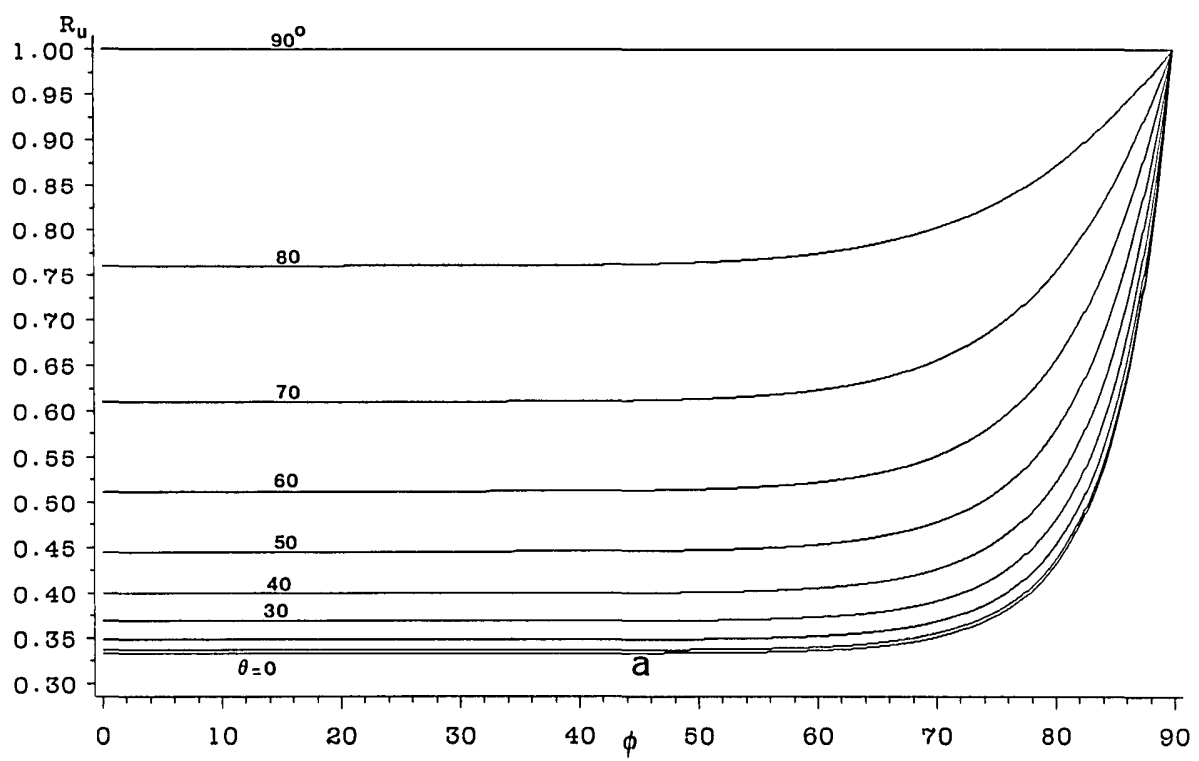

Fig. 4. Family of maximally flat unpolarized-light reflectance $R_{u}$ versus the angle of incidence $\phi$ that correspond to 10 discrete points on the HIB of the limaçon of Pascal in Fig. 1 at angles $\theta$ ranging from $0^{\circ}$ to $90^{\circ}$ in equal steps of $10^{\circ}$. Curve a corresponds to a dielectricdielectric interface with relative refractive index $N=2+\sqrt{3}=3.73205$ (point A in Fig. 1). 


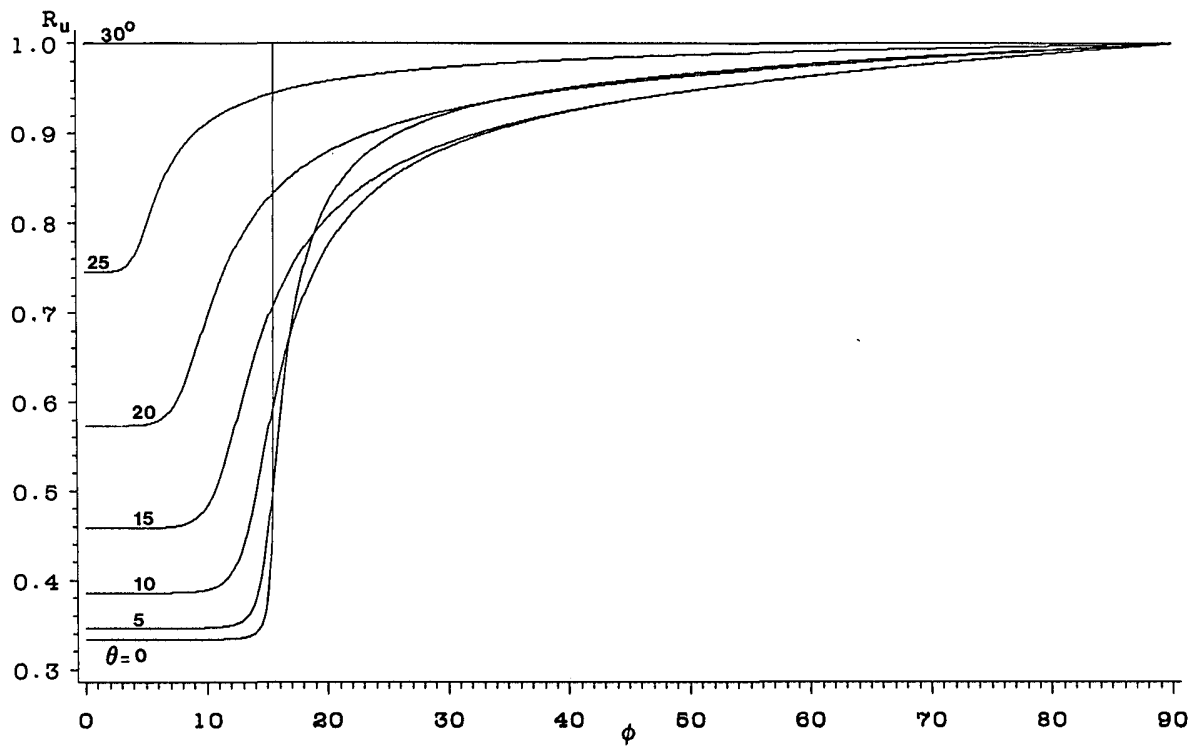

Fig. 5. Unpolarized-light reflectance $R_{u}$ versus the angle of incidence $\phi$ for seven discrete points on the LIB of the limaçon of Pascal in Fig. 1 at angles $\theta$ ranging from $0^{\circ}$ to $30^{\circ}$ in equal steps of $5^{\circ}$. This is the domain of internal reflection and fractional optical constants $n$ and $k$.

Eq. (41) can be solved for $\phi$ as its only unknown by numerical iteration.

Table 1 shows some results for GaAs in the visible. Listed are the photon energy $h \nu$ (in electron volts), the wavelength $\lambda$ (in nanometers), the real and the imaginary parts $\epsilon_{r}$ and $\epsilon_{i}$ of complex $\epsilon$, the angle of incidence of minimum unpolarized-light reflectance $\phi_{\text {umin }}$ obtained by solving Eq. (41), and the associated minimum reflectance $R_{\text {umin }}$.

For interfaces between dielectric media, $\epsilon$ is real, $r_{\nu}{ }^{*}=$ $r_{\nu}$, and Eq. (40) leads to

$\epsilon(\epsilon \cos \phi-X)(\cos \phi+X)^{3}$

$$
+(\cos \phi-X)(\epsilon \cos \phi+X)^{3}=0 .
$$

By expanding the terms in Eq. (42) and replacing every $X^{2}$ and $X^{4}$ by $\left(\epsilon-\sin ^{2} \phi\right)$ and $\left(\epsilon^{2}-2 \epsilon \sin ^{2} \phi+\sin ^{4} \phi\right)$, respectively, we obtain the following equation that is linear in $X$

$$
\left(\epsilon^{2}-6 \epsilon+1\right)(X \cos \phi)=(\epsilon+1)\left[-(\epsilon+1) \sin ^{2} \phi+2 \epsilon\right] \text {. }
$$

Finally, squaring both sides of Eq. (43) and substituting

$$
u=\sin ^{2} \phi
$$

lead to a quadratic equation in $u$ :

$$
a u^{2}+b u+c=0,
$$

in which $a, b$, and $c$ in their most reduced form are given by

$$
\begin{aligned}
& a=16 \epsilon, \\
& b=(\epsilon+1)\left(\epsilon^{2}-14 \epsilon+1\right), \\
& c=-\epsilon\left(\epsilon^{2}-14 \epsilon+1\right) .
\end{aligned}
$$

The solution of Eq. (45) for $u$ is of course explicit:

$$
u=(-b \pm d) / 2 a \text {, }
$$

where

$$
\begin{aligned}
d & =\left(b^{2}-4 a c\right)^{1 / 2} \\
& =\left(\epsilon^{2}-6 \epsilon+1\right)\left(\epsilon^{2}-14 \epsilon+1\right)^{1 / 2} .
\end{aligned}
$$

Equation (47) yields an acceptable root for $u, 0 \leq u<1$, when

$$
\epsilon \geq(2+\sqrt{3})^{2}=13.9282 \text { or } \epsilon \leq(2-\sqrt{3})^{2}=0.0718 \text {. }
$$

The angle of minimum $R_{u}$ is determined by

$$
\phi_{\text {umin }}=\arcsin u^{1 / 2} \text {. }
$$

The foregoing explicit analytic solution for the angle of incidence of minimum reflectance of a dielectric interface for incident unpolarized light is, to my knowledge, new, although the problem of defining this angle was considered in 1919 by Jentzsch-Gräfe. In this early paper ${ }^{7}$ an analytic relation between the angles of incidence and refraction that holds at the minimum is found. This relation, in addition to Snell's law, provides an implicit solution for $\phi_{\text {umin }}$.

In Appendix A we consider the interesting related problem of determining the refractive index of a high-index dielectric medium from the angle of incidence of minimum unpolarized-light reflectance.

For illustration, Fig. 6 shows a family of $R_{u}$-versus- $\phi$ curves for dielectric interfaces with real $N$ from 1.5 to 6.0 in steps of 0.5. Superimposed upon Fig. 6 is the MFR curve for $N=3.73205$, which is indicated by the pluses

Table 1. Angle of Incidence $\phi_{\text {umin }}$ of Minimum Reflectance for Unpolarized Light Incident at the Air-GaAs Interface ${ }^{a}$

\begin{tabular}{cccccc}
\hline$h \nu(\mathrm{eV})$ & $\lambda(\mathrm{nm})$ & $\epsilon_{r}$ & $\epsilon_{i}$ & $\phi_{\text {umin }}\left({ }^{\circ}\right)$ & $R_{\text {umin }}$ \\
\hline 1.8 & 689 & 14.303 & 1.143 & 42.020 & 0.3393 \\
2.0 & 620 & 14.994 & 1.637 & 51.689 & 0.3485 \\
2.2 & 564 & 16.028 & 2.215 & 58.443 & 0.3605 \\
2.4 & 517 & 17.544 & 3.120 & 63.898 & 0.3755 \\
2.6 & 477 & 19.888 & 4.842 & 68.758 & 0.3946 \\
2.8 & 443 & 23.610 & 9.829 & 73.574 & 0.4218 \\
\hline
\end{tabular}

${ }^{a} \phi_{\text {umin }}$ is obtained by a numerical iterative solution of Eq. (41). $R_{\text {umin }}$ is the associated minimum reflectance. $h \nu$ and $\lambda$ are the photon energy and the wavelength of light, respectively. $\epsilon_{r}$ and $\epsilon_{i}$ are the real and the imaginary parts of the complex relative dielectric function $\epsilon$ of $\mathrm{GaAs}$ as obtained from Ref. 6 . 


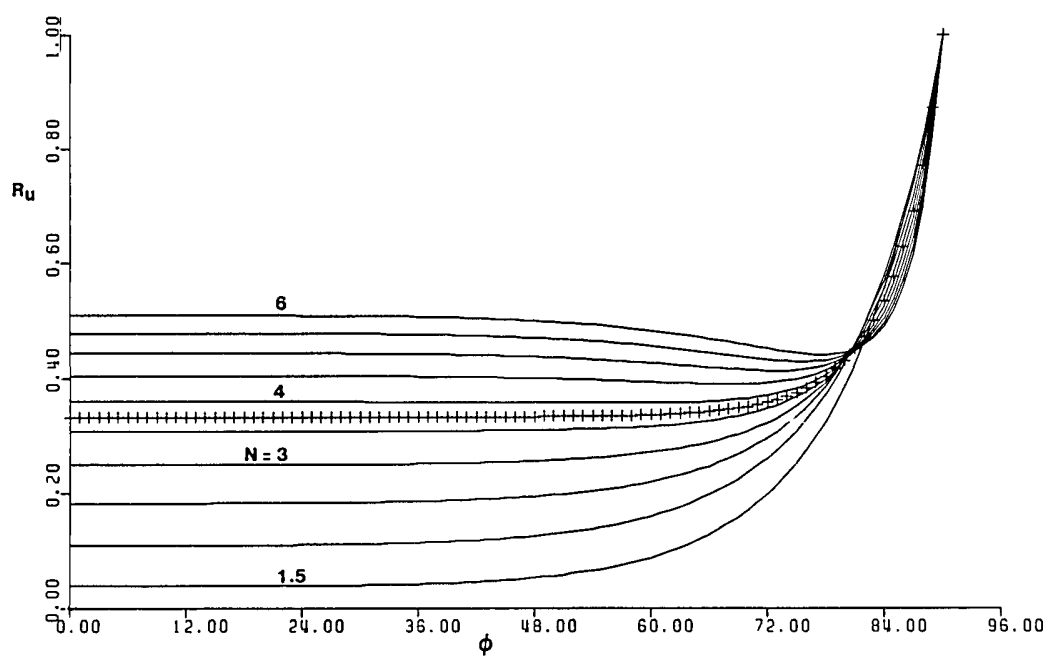

Fig. 6. Unpolarized-light reflectance $R_{u}$ versus the angle of incidence $\phi$ of dielectric-dielectric interfaces with relative refractive indices ranging from 1.5 to 6.0 in steps of 0.5 . Also indicated is the maximally flat curve for $N=3.73205$, which is marked with the pluses ( + ).

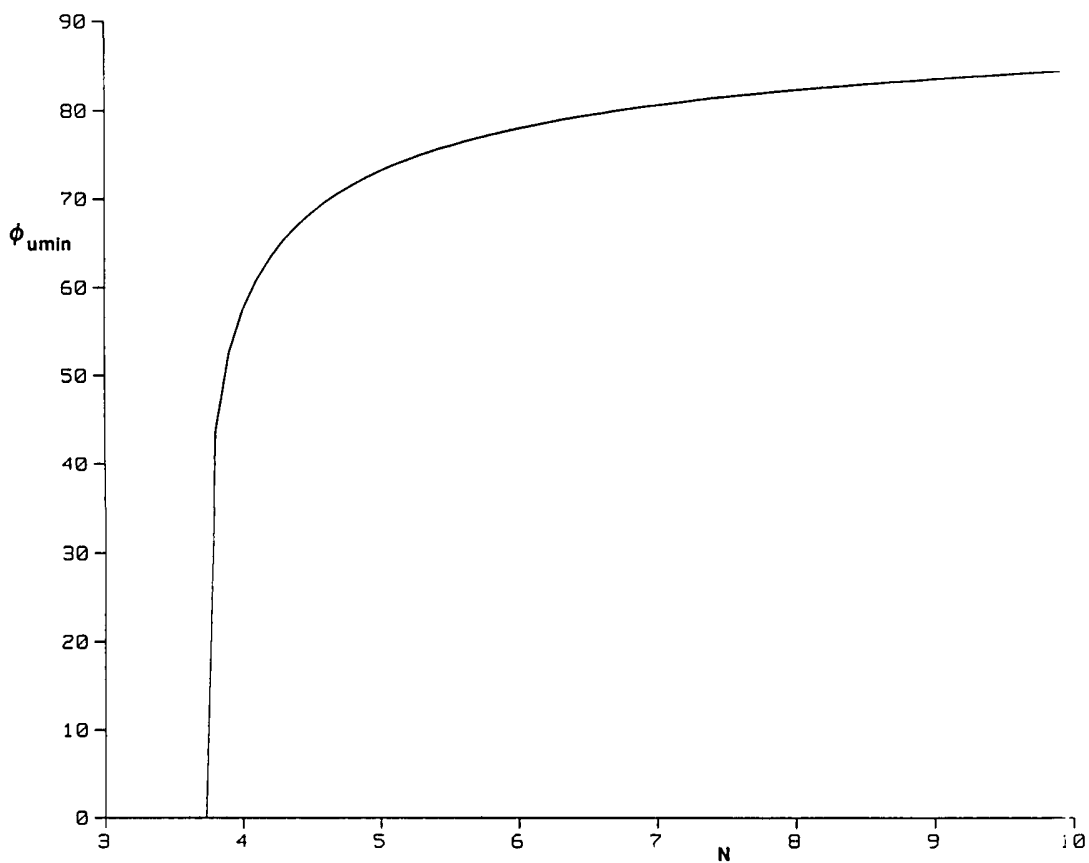

Fig. 7. Angle of incidence of minimum reflectance for incident unpolarized light $\phi_{\text {umin }}$ as a function of the relative refractive index $N>$ 3.73205 of a dielectric interface. $\phi_{\text {umin }}$ is calculated by using Eqs. $(46)-(50)$.

(t). At least to the eye, the curves nearest the MFR curve, for $N=3.5$ and $N=4.0$, appear to be equally flat. $^{8}$ This illustrates a general insensitivity of the flatness of the reflectance curve with respect to small changes of $N$ in the neighborhood of a point on the limaçon of Pascal (in this case point A in Fig. 1).

Figure 7 shows $\phi_{\text {umin }}$ as a function of $N \geq 3.73205$. The initial steep rise of $\phi_{\text {umin }}$ with $N$ is typical of the variation of this angle with radial distance starting from an arbitrary point on the limaçon of Pascal.

\section{CONCLUSIONS}

The conditions for monotonic and nonmonotonic variations of the unpolarized-light reflectance of an interface with incidence angle have been determined exactly. In particular, if the complex relative refractive index of an interface (i.e., the ratio of the complex refractive index of the medium of refraction to the real refractive index of the transparent medium of incidence) $N=\eta \exp (j \theta)$ is such that $2 \cos \theta-\sqrt{3} \leq \eta \leq 2 \cos \theta+\sqrt{3}$, the reflectance is monotonic with angle; otherwise the reflectance exhibits a minimum at oblique incidence. The boundary line separating the regions of monotonic and nonmonotonic behaviors is a limaçon of Pascal. New equations that locate the angle of incidence of minimum reflectance for incident unpolarized or circularly polarized light are derived. For a dielectric interface, the equation is quadratic in the sine squared of the incidence angle with an exact explicit solution. 


\section{APPENDIX A: REFRACTIVE INDEX OF A HIGH-INDEX SUBSTRATE FROM THE ANGLE OF INCIDENCE OF MINIMUM REFLECTANCE FOR INCIDENT UNPOLARIZED LIGHT}

The refractive index $N$ of a dielectric substrate is easily determined in terms of the Brewster angle $\phi_{B}$ of minimum (zero) reflectance for incident $p$-polarized light by

$$
N=\tan \phi_{B}
$$

Suppose that the incident light is unpolarized (or circularly polarized) and that the refractive index of the substrate is sufficiently high for the reflectance to have a resolvable minimum at an angle $\phi_{\text {umin }}$ that is measured. How can $N$ be determined from $\phi_{\text {umin }}$ ? The answer resides in Eqs. (45) and (46).

Substitution of the coefficients $a, b$, and $c$ from Eqs. (46) into Eq. (45) gives a cubic equation in $\epsilon$ :

$$
q_{3} \epsilon^{3}+q_{2} \epsilon^{2}+q_{1} \epsilon+q_{0}=0
$$

in which

$$
\begin{aligned}
& q_{0}=u, \\
& q_{1}=16 u^{2}-13 u-1, \\
& q_{2}=14-13 u, \\
& q_{3}=u-1,
\end{aligned}
$$

and $u=\sin ^{2} \phi_{\text {umin }}$. Cubic equation (A2) with coefficients given by Eqs. (A3) can be solved exactly ${ }^{9}$ for $\epsilon$, and only a positive real root is acceptable. $N$ is subsequently determined by $N=\epsilon^{1 / 2}$.
As an example, suppose that $\phi_{\mathrm{umin}}=45^{\circ}$, so that $u=$ $1 / 2$. The corresponding cubic equation becomes $\epsilon^{3}-$ $15 \epsilon^{2}+7 \epsilon-1=0$. The only acceptable root of this cubic is $\epsilon=14.5228$; hence $N=3.811$. The other two roots of this cubic are complex conjugates and hence unacceptable.

\section{ACKNOWLEDGMENTS}

I thank Abdalla Alnajjar and Taline Yeretzian for their assistance with some of the computations.

\section{REFERENCES AND NOTES}

1. See, for example, M. Born and E. Wolf, Principles of Optics (Pergamon, New York, 1975), Sec. 1.5.

2. R. M. A. Azzam, "Stationary property of normal-incidence reflection from isotropic surfaces," J. Opt. Soc. Am. 72, 11871189 (1982).

3. See, for example, W. H. Beyer, ed., Standard Mathematical Tables, 28th ed. (CRC Press, Boca Raton, Fla., 1987), p. 220.

4. H. B. Holl, "Specular reflection and characteristics of reflected light," J. Opt. Soc. Am. 57, 683-690 (1967).

5. R. M. A. Azzam and A. M. Alnajjar, "Bare and thin filmcoated substrates with maximally flat reflectance vs angle of incidence curve for incident unpolarized light," in Optical Society of America Annual Meeting, Vol. 18 of 1989 OSA Technical Digest Series (Optical Society of America, Washington, D.C., 1989), paper TUR5.

6. E. D. Palik, ed., Handbook of Optical Constants of Solids (Academic, New York, 1985). See pp. 565, 436-439, and 793 for the cited optical constants of $\mathrm{Si}, \mathrm{GaAs}$, and $\mathrm{NaCl}$, respectively.

7. F. Jentzsch-Gräfe, "Minimalstellen der regulären Reflexion," Verh. Dtsch. Phys. Ges. 21, 361-368 (1919).

8. Other criteria for flatness, besides that specified by Eq. (9), can be considered. For example, one may wish to maximize the range of incidence angle over which the fractional deviation of reflectance from its normal-incidence value, $\mid R_{u}-$ $R_{0} \mid / R_{0}$, is less than a prespecified small limit or tolerance (e.g., 0.001).

9. See, for example, J. J. Tuma, Engineering Mathematics Handbook, 3rd ed. (McGraw-Hill, New York, 1987), p. 7. 